\title{
An Analysis Of Bottleneck Technology By Using Experiments And CAE -Example Of The Automotive Transaxle Oil Seal Leakage-
}

Takahiro Ito, Aoyama Gakuin University, Japan

Masatoshi Sato, Aoyama Gakuin University, Japan

Manabu Yamaji, Waseda University, Japan

Kakuro Amasaka, Aoyama Gakuin University, Japan

\begin{abstract}
This research aims to clarify the technological mechanism by using experiment and CAE (Computer Aided Engineering). Concretely, we take up the automotive transaxle oil seal leakage. To clarify an unknown mechanism, the dynamic behavior on the surface of the seal lip in the drive shaft high-speed rotation was observed with the visualization device. The authors thus achieved the desired results.
\end{abstract}

Keywords: the automotive transaxle oil leakage; the visualization experiment; CAE

\section{INTRODUCTION}

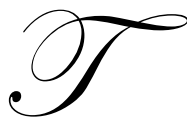

he automotive industry is engaging in a "global production strategy for simultaneous achievement of QCD (quality, cost and delivery)" aiming to achieve "worldwide uniform quality and production at optimum locations", in an effort to prevail and survive in the "worldwide quality competition". It is now necessary to reform the conventional development and production method. In other words, there will be a conversion from the so-called "development through real object confirmation and improvement" to "prediction evaluation oriented development" (Amasaka 2008).

In order to realize this development, it is important to raise the ratio of the application of CAE (Computer Aided Engineering) by utilizing numerical simulation. CAE needs to explore the technological mechanism to be useful, and to create the generalized model. Therefore, the authors sought to contribute to the creation of the generalized model by finding solutions to cases with unknown mechanisms.

In this research, the viewpoint is to grasp the dynamic behavior of the technical problem by using experiments as the empirical approach and the numerical simulation.

This study aims to clarify the technological mechanism by using experiment and CAE. Concretely, we take up the automotive transaxle oil seal leakage. As the result, the difference was seen by the material and the shape of the oil seal. The desired results were thus achieved.

\section{EXPECTATIONS FOR AUTOMOTIVE DEVELOPMENT PRODUCTION AND SIMULATION TECHNOLOGY}

Resent Japanese enterprises have been promoting global production to realize uniform quality worldwide and production at optimal locations for severe competition. The mission of the automotive manufacturers in this rapidly changing management technology environment, is to be prepared for the "worldwide quality competition", so as not to be pushed out of the market and to establish a new management technology model which enables them to offer highly reliable products of the latest design that are capable of enhancing the value to the customer. 
In the area of management technology for the development and production processes that is being considered here, excessive repetition of "prototyping, testing, and evaluation" has been carried out for the purpose of preventing the "scale-up effect" in the bridging stage between testing and mass production. This has resulted in an increase in the development period and cost. Therefore, it is now necessary to reform the conventional development and production method. More specifically, it is increasingly vital to realize the "simultaneous achievement of QCD" (Quality, Cost and Delivery) that satisfies the requirements of developing and producing high quality products, while also reducing the cost and development period through incorporation of the latest simulation technology "CAE" (Computer Aided Engineering) and statistical science called SQC (Statistical Quality Control).

In the vehicle development process employed in the past, after completing the designing process, problem detection and improvement were repeated mainly through the process of prototyping, testing, and evaluation. In some current automotive development, a prototype of a vehicle body is not manufactured in the early stage of development due to the utilization of $\mathrm{CAE}$ and SE (simultaneous engineering) activities, and therefore the development period has been substantially shortened (first from four years to two years, and then to one year at present).

Given this background, therefore, the conventional development process of repeated evaluation using prototypes is no longer capable of handling this task. Collaboration between CAE and SE activities, which are now faster and more precise, will be indispensable for fully utilizing the accumulated knowledge database. As discussed so far, expectations are high for the realization of super short-term development, which would be done through utilization of CAE. In other words, there will be a conversion from the so-called "development through real object confirmation and improvement" to "prediction evaluation oriented development" (Amasaka 2008, Yamaji et al. 2008).

\section{THE NECESSITY OF CLARIFYING TECHNOLOGICAL MECHANISMS}

The hardware-software environment surrounding CAE has evolved. Though technological problems have been understood and general solutions have been derived and reflected in CAE analysis software, research findings that boost the credibility of CAE have still not been incorporated fully enough into the development design process. Therefore, because predicted CAE results are not reproduced experimentally and CAE experiments must be redone, reducing time to market and development cost currently remains more the ideal than the reality. (Amasaka 2008)

To revolutionize the design of forecast evaluation systems that effectively use CAE using the design of actual confirmation improvement systems, products must be evaluated and analytical CAE results must be guaranteed. It is important to outline a failure mechanism that captures the true reason for problems. The authors have attempted to clarify one pressing problem experienced by a worldwide automaker. The problem is an unknown mechanism causing an oil seal leakage problem on the surface of the drive shaft during high-speed rotation.

\section{DYNAMIC BEHAVIOR VISUALIZATION EXPERIMENT}

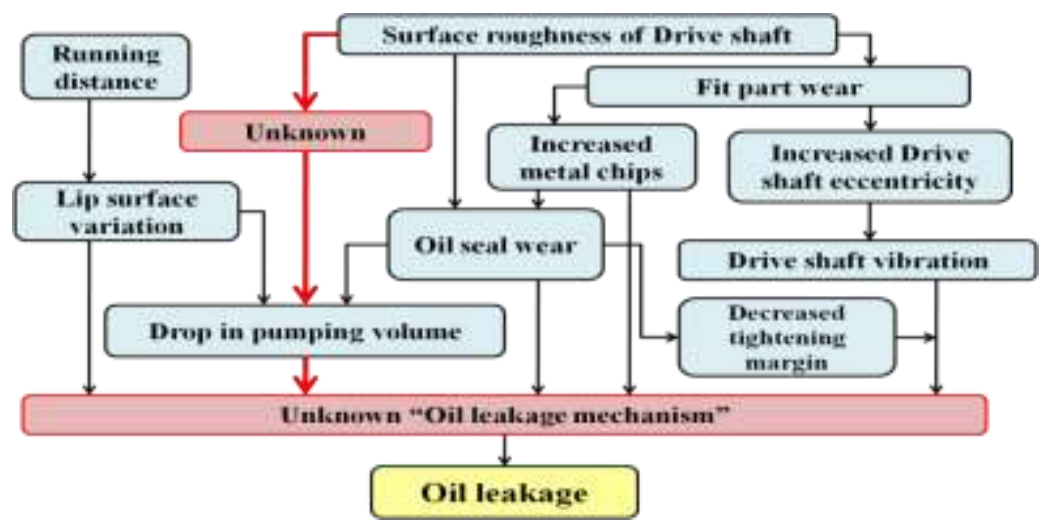

Figure 1: Estimation of the Oil Leakage Mechanism 
The study on the oil leak mechanism involved looking at different issues affecting the leak through several studies, as shown in Figure 1. Causal relationships for the oil leakage were investigated using a relationship drawing to arrange related factors and knowledge previously obtained. Because the oil leakage mechanism is uncertain, the generation route could not be clarified. Locations where it is necessary to make the dynamic behavior visible are specified, and experiments were conducted on devices to make it visible (Amasaka et al. 2001, 2002).

\section{Visualization Device}

In order to visualize the dynamic behavior of the oil seal lip, a device to make oil seal visible as shown in Figure 2 was developed. The oil seal was reproduced as shown in the figure, and when soaked in lubricant in a similar way, changed into a glass axis that rotated the drive shaft eccentrically via the spindle motor. Operation in an actual vehicle was then reproduced with the transaxle.

The effect of sealing the oil seal lip was visualized using an optical fiber. The sliding side observation experiment used three types of seals made of different materials and shapes, and identified the status of sliding surfaces and their relationship to stopping the leakage. The experiment helped in gaining clear knowledge about the unknown mechanism (Amasaka 2003).

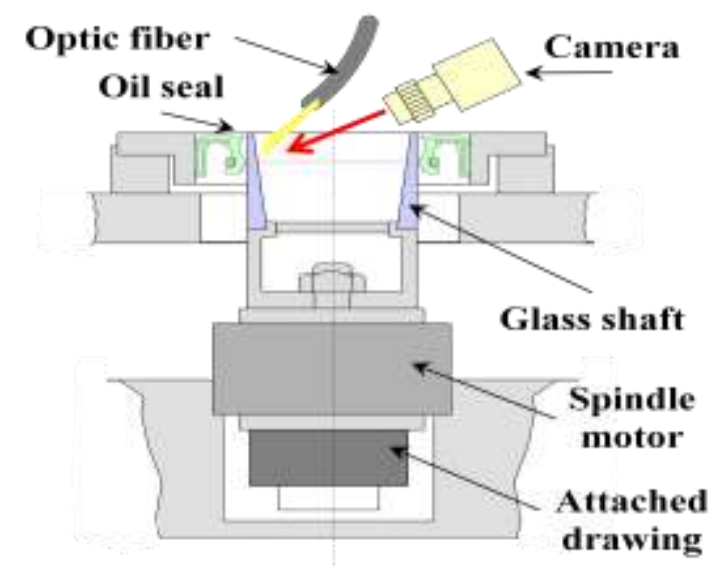

Figure 2: Outline of device by visualization

\section{Sliding surface visualization experiment}

The sliding side observation experiment was conducted using three types of seals made of different materials and shapes. Here, two seal characteristic values are defined: (1) $X_{G}$ and (2) $A_{R}$ as shown in Figure 3. XG is the distance that the true contact area center of gravity is biased from the sliding side center to either the oil side $\left(1>X_{G}>0\right)$ or the atmospheric side $\left(-1<X_{G}<0\right)$; it is the characteristic value that shows the extent true contact area distribution bias axially. $\mathrm{X}_{\mathrm{G}}$ can be interpreted as the maximum pressure position of the contact pressure distribution. $A_{R}$ is a characteristic value indicating roughness on the seal sliding side level, and shows the proportion of the visible touch area that is the real contact area.

Table 1 shows the characteristic seal values and the outcome of the experiments for the three types. In addition, leakage stopping can be classified according to both characteristic values by looking at the relationship between these two characteristic values and the observed state of the leakage stopping as shown in Table 1. Sealing is achieved only in Seal 1 in the table, and sliding surfaces show the characteristic values $X_{G}>0$ and $A_{R}<0.05(5 \%)$. It is evident that the unique sliding side structure to ensure sealing has minute surface roughness (existence of minute projections) and the true contact area is biased towards the oil side (Sato et al. 1999, 2003, Kameike et al. 2000). 


\section{Oil Side $\quad$ (b:Contact Width)}

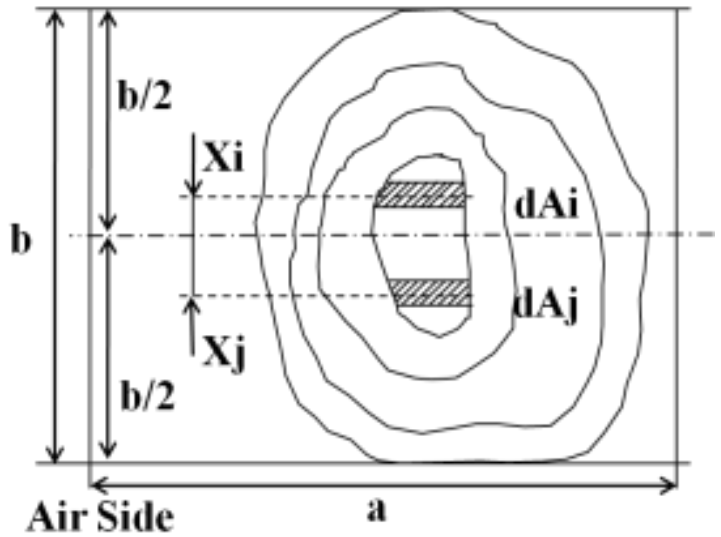

Figure 3: Pattern diagram of the sliding side

\begin{tabular}{|c|c|c|c|}
\hline & Type 1 & Type 2 & Type 3 \\
\hline $\mathbf{X}_{\mathrm{G}}$ & 0.02 & 0.47 & 0.04 \\
\hline $\mathrm{A}_{\mathrm{R}}$ & 0.14 & 0.05 & -0.21 \\
\hline result & Sealing & Leakage & Leakage \\
\hline
\end{tabular}

Table 1: Outcome of the experiments

$$
\begin{aligned}
& X_{a}=\frac{\sum d A i\left(\frac{X_{i}}{b / 2}\right)-\sum d A j\left(\frac{X_{j}}{b / 2}\right)}{\sum d A i+\sum d A j} \\
& A_{k}=\frac{\text { The real contact area }}{\text { The visible touch area }}=\frac{(\text { Shaded area })}{(a \times b)}
\end{aligned}
$$

\section{Sliding surface basic structure model}

The condition of the sliding side, which became clear through visibility experiment, is used as a basic component to generate the basic sliding surfaces structural model as shown in Figure 4. This model shows the minute roughness that existed on the sliding surfaces by statistically approximating a central projection.

Sealing of the entire sliding side is achieved when the intake and outtake quantity of oil is QAA'> QBB', due to minute projection AA' acting as a wedge being larger than minute projection BB'. In addition, this oil flow works to prevent wear by forming a circulation pattern on the sliding side and separating the two sliding surfaces (Sato et al. 1999, 2003, Kameike et al. 2000).

\section{OIL SEAL SIMULATION}

\section{Technological Elements of the Oil Seal Simulator}

The technological elements used to do numerical analysis must be selected using findings obtained from the visibility experiment. In general, because CAE first establishes the problem to be solved and models the problem using formulas, a computer is used as an initial method and to analyze the model. The algorithm (calculation 
routine) is then used as an analytical method. Validity, coverage, and the performance of this algorithm stem from the theory, and computer technology is what actually carries out the calculation function (calculation technique) (Ueno et al. 2008).

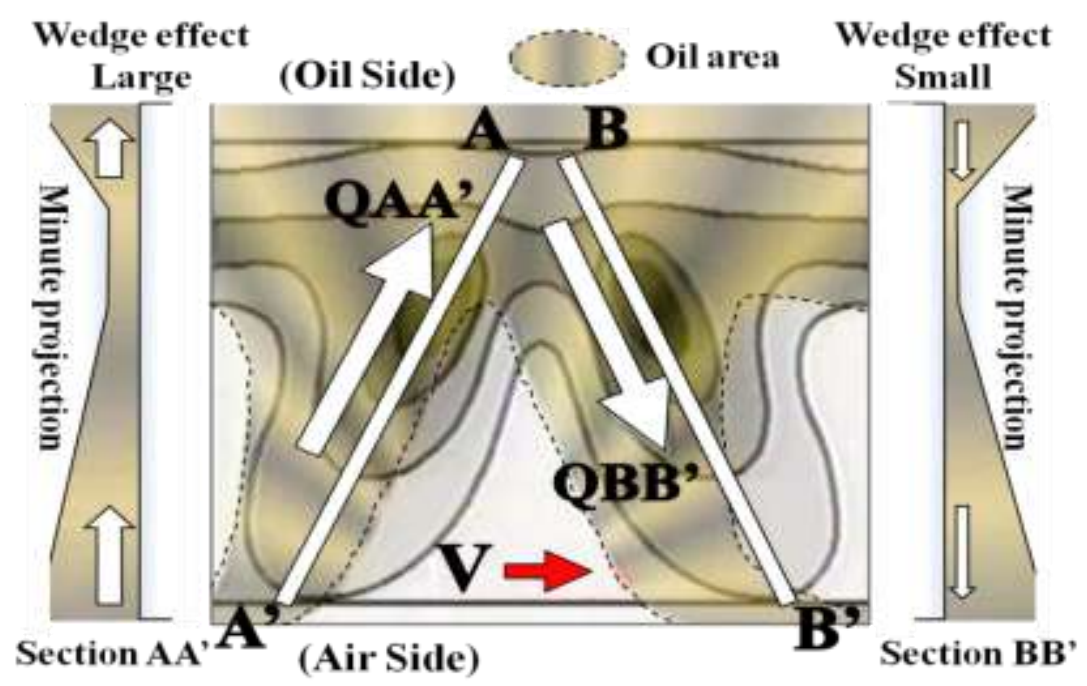

Figure 4: Sliding surface basic structure model

It is important to appropriately select technological elements such as establishing the problem, modeling, the algorithm, theory, and computer technology to obtain high reliability when analyzing CAE. Organically relating these technological elements result in successful CAE analysis. Figure 5 shows the technological elements that the oil seal simulator should have. In handling the oil leakage phenomenon, the problem is grasping the pump volume and lip side pressure distribution that directs the behavior and the oil circulation pattern on the minute projection area of sliding surfaces.

The fluid resistance model, the contact model, and the material component rule model are used to solve these problems. The finite element method and numeric fluid is analyzed as a convenient algorithm. The Reynolds equation, Soft Elasto-Hydrodynamic Lubrication, and Navier-Stokes equation are appropriate theoretical formulas. Accuracy is ensured, and the time integration method, space difference method, and procession method are used as computer technologies to calculate in a realistic timeframe. Each of the above elements is used to construct the oil seal simulator.

\section{Oil Seal Simulation Results}

The authors utilized the technological elements as shown in Figure 5 to execute the oil seal simulation. The numerical simulation intended for the oil slick on the sliding side was done for the three seal types used in the visibility experiment to understand oil behavior and pump volume on the minute projection area that was the problem.

Table 2 shows the results of making the analytical model as shown in Figure 6 from the basic sliding surfaces structural model in Figure 4 above. This was done given the rotational speed and other conditions of the shaft, and calculating the quantity of oil flowing on the oil side and atmospheric side. Results similar to the visibility experiment were reported. In addition, the following findings were obtained by numerical analysis:

(1) For seals that successfully sealed up, inlet flow > outlet flow, creating a circulation pattern.

(2) For leaking seals, a great deal of oil was flowing on the atmospheric side. 


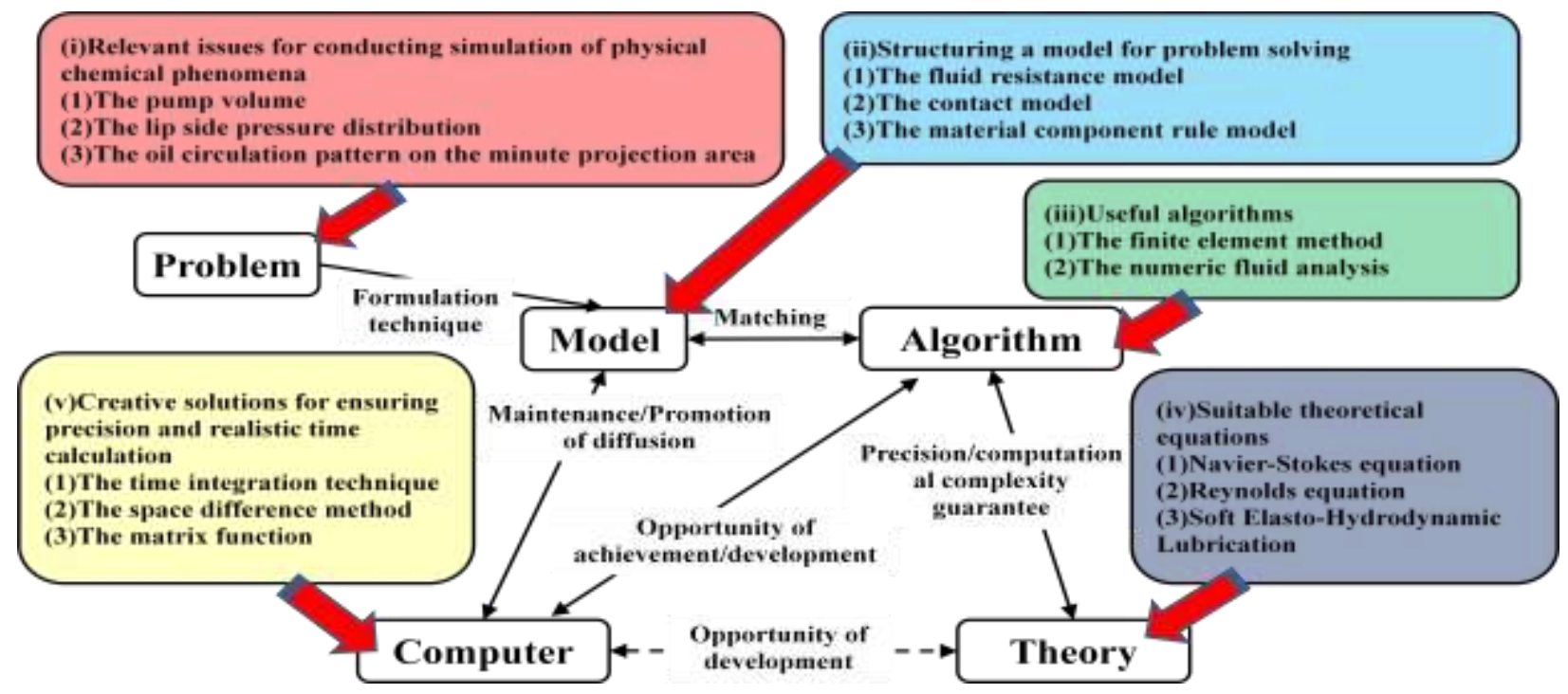

Figure 5: Technological Elements of the Oil Seal Simulator

Table 2: Analysis result

\begin{tabular}{|c|c|c|c|}
\hline Pump Volume & Type 1 & Type 2 & Type 3 \\
\hline Oil Side & 28.57 & 2.21 & 19.7 \\
\hline Air Side & 6.84 & -4.24 & -22.6 \\
\hline Total & 35.41 & -2.03 & -2.89 \\
\hline
\end{tabular}

Seal type 1

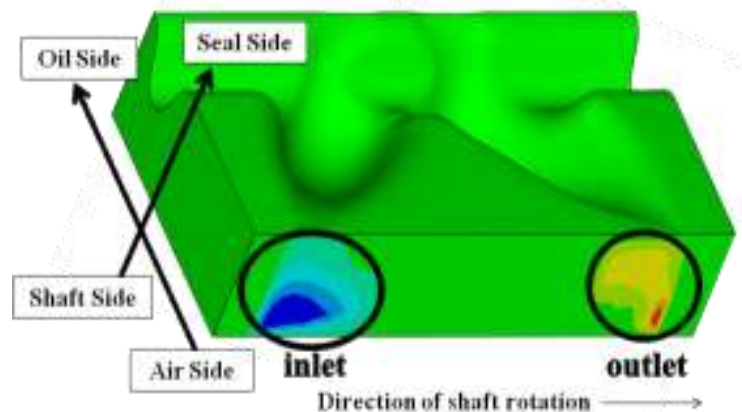

Seal type 2

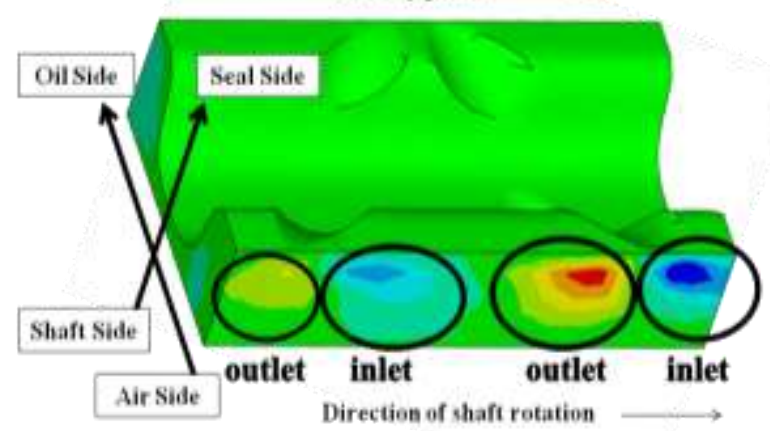

Seal type 3

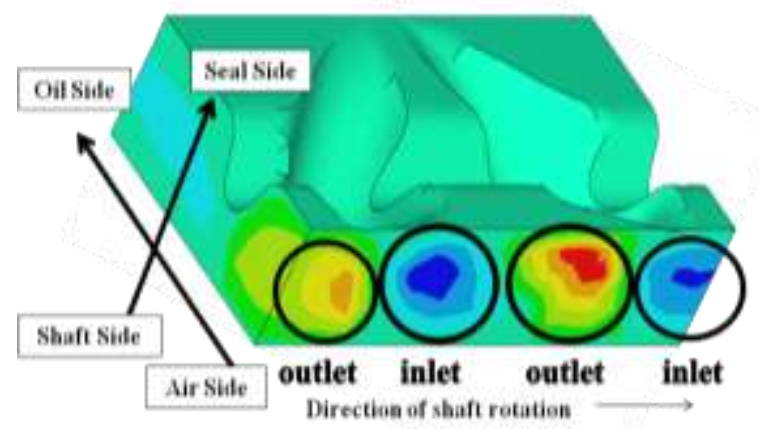

Figure 6: Analysis Result 


\section{CONCLUSION}

The visibility device experiment together with CAE made it possible to identify the following mechanisms related to oil seal leaks in the vehicle drive train: (1) the behavior of oil on the sliding side, (2) the characteristic values of the seals, and (3) volume of leakage stopping pumps.

\section{AUTHOR INFORMATION}

Takahiro Ito is a graduate student of the College of Science and Engineering at Aoyama Gakuin University.

Masatoshi Sato is received his Bachelor of Engineering degree from the College of Science and Engineering at Aoyama Gakuin University.

Manabu Yamaji is a researcher in the Advanced Research Center for Human Sciences at Waseda University, Japan. He received his Master of Engineering degree in Graduate School of Information Systems at University of Electro-Communications in 1999. His current research and teaching interests are in the general area of production engineering. In particular, he is interested in Total Quality Management, Computer Aided Engineering.

Kakuro Amasaka is a Professor in the College of Science and Engineering at Aoyama Gakuin University, Japan. He received his Ph.D. degree in Precision Mechanical and System Engineering, Statistics and Quality Control at Hiroshima University in 1997. His current research and teaching interests are in the general area of production engineering. In particular, he is interested in New JIT. He is a member of POMS and EurOMA.

\section{REFERENCES}

1. M. Lopez, K. Nakamura, K. Seki (1997), A study on the sealing characteristics of lip seals with helical ribs, in the 15th International Conference of British Hydromechanics Research Group Ltd. Fluid Sealing, 1-11.

2. K. Amasaka, S. Osaki (2001), A Demonstrative Study on High Reliability of Drive System Design, John Wiley \& Sons, Inc.

3. K. Amasaka, S. Osaki (2002), A Reliability of Oil Seal for Transaxle - A Science SQC Approach in Toyota-, Case Studies in Reliability and Maintenance by Wallace R. Blischke and D. N. P. Murthy, to be published by John Wiley \& Sons, Inc., 571-581.

4. K. Amasaka (2003), A dual total task management team involving both Toyota and NOK, in the Group Technology/ Cellular Manufacturing World Symposium, Columbus Ohio, 265-270.

5. K. Amasaka (2008), An Integrated Intelligence Development Design CAE Model Utilizing New JIT: Application to Automotive High Reliability Assurance, Journal of Advanced Manufacturing Systems, 7(2), 221-241

6. $\quad$ K. Nakamura (1987), Sealing mechanism of rotary shaft lip-type seals, Tribology International. 20(2), 90101.

7. M. Kameike, S. Ono and K. Nakamura (2000), The helical seal: Sealing concept and rib design, Sealing Technology, 77, 7-11.

8. M. Yamaji, K. Amasaka (2008), CAE Analysis Technology for Development Design Utilizing Statistical Sciences, The Open Industrial and Manufacturing Engineering Journal, 1, 1-8.

9. T. Ueno, M. Yamaji, H. Tsubaki, K. Amasaka (2009), Establishment of Bolt Tightening Simulation System for Automotive Industry Application of the Highly Reliable CAE Model, International Journal of Electronic Business Management, 8(5), 57-67.

10. Y. Sato, A. Toda, S. Ono, K. Nakamura (1999), A study of the sealing mechanism of radial lip seal with helical ribs -measurement of the lubricant fluid behavior under sealing contact, SAE Technical Paper Series.

11. Y. Sato, K. Seki, J. Sugimura, Y. Yamamoto (2003), Experiments and simple modeling of hydrodynamic lubrication in radial shaft seals, Proc. $17^{\text {th }}$ Int. Conf. Fluid Sealing, BHR Group, 139-156. 
NOTES 